We also observed an increased expression of PDL2 on RA-

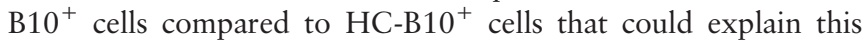
effect on Th1 differentiation.

Conclusions IL-10 secretion can be an interesting marker to define Breg subsets acting on regulatory $\mathrm{T}$ cell differentiation. Thus, increasing the number of $\mathrm{B}^{+} 0^{+}$cells, seems a promising therapeutic strategy especially in patients who lack the most of Treg cells. Additional markers are needed to define which subset of Breg cells can control inflammatory response. The role of PD1/PDL2 in $\mathrm{B}^{+} 0^{+}$cell function might also open new insights.

Disclosure of interest None declared

\section{CHARACTERISING THE ROLE OF NET-DERIVED IL-33 IN SLE PATHOGENESIS}

1,2S Georgakis* ${ }^{1,2}$ G Papadaki, ${ }^{3}$ I Gergianaki, ${ }^{3} \mathrm{C}$ Adamichou, ${ }^{3} \mathrm{~S}$ Polia, ${ }^{1,3} \mathrm{P}$ Sidiropoulos, ${ }^{4} \mathrm{P}$ Verginis, ${ }^{1,2} \mathrm{~K}$ Gkirtzimanaki, ${ }^{1,3} \mathrm{G}$ Bertsias. 'Rheumatology, Autolmmunity and Inflammation Laboratory, Medical School, University of Crete; ${ }^{2}$ Institute of Molecular Biology and Biotechnology, Foundation of Research and Technology - Hellas; ${ }^{3}$ Rheumatology Department, University General hospital, Heraklion; ${ }^{4}$ Biomedical Research Foundation, Academy of Athens, Athens, Greece

\subsection{6/annrheumdis-2018-EWRR2018.3}

Introduction Interleukin (IL)-33 is a cell necrosis-derived alarmin with immunostimulatory properties which depends on the context of immune cells and the inflammatory milieu. ${ }^{1,2}$ In Systemic Lupus Erythematosus (SLE), extracellular DNA (as in extracellular chromatin traps [NETs] or immune complexes [ICs]) combined with alarmins stimulate innate immunity receptors (such as Toll-like receptors [TLRs]) and the production of IFNa by plasmatocytoid dendritic cells (pDCs). ${ }^{3,4}$

Objectives We hypothesised that bioactive NET-derived isoforms of IL-33 might augment the interferogenic potential of extracellular DNA in SLE.

Methods Peripheral blood polymorphonuclear cells (PMNs) were isolated from healthy and SLE individuals and their ability to form IL33-decorated NETs was assessed by confocal microscopy. NET-supernatants from untreated and IC-treated SLE PMNs were administrated to healthy pDCs and type I IFN production was monitored by qPCR and ELISA. The contribution of IL-33 in the interferogenic capacity of NETs was addressed by pre-treating $\mathrm{pDCs}$ with a specific $\mathrm{mAb}$ against IL33-receptor (anti-ST2L). Western blotting of SLE and healthy PMNs was used to detect different isoforms of IL-33. Results Spontaneous-released NETs from peripheral blood PMNs of active SLE patients were decorated with IL-33 to a larger extent as compared to healthy PMN NETs. Treatment of SLE PMNs with ICs led to the release of NETs with extended IL-33 decoration and enhanced interferogenic capacity. IL33 receptor blockade significantly decreased the type I IFN response of NET-treated control pDCs. DNase treatment of NETs to disrupt their structure resulted in reduced interferogenic capacity and abrogated the pro-inflammatory effect of IL-33. Finally, western blotting on IC-mediated SLE NETs revealed the presence of cleaved isoforms of IL-33 which closely resemble the previously characterised highly bioactive isoforms of our protein of interest. Experiments are underway to explore the possible role of NETbound proteases in IL-33 activation.

Conclusions NET-derived IL-33 is a novel mediator of the nucleic acid-driven aberrant type I IFN response which exacerbates SLE disease. NET structure may be crucial in regulating the bioactivity of IL-33.

\section{REFERENCES}

1. Foo Yew Liew, et al. Intereleukin-33 in health and disease. Nature Reviews 2016.

2. Lefrancais E. IL-33 is processed in mature bioactive isoforms by neutrophil elastase and cathepsin G. PNAs 2012

3. Garcia Romo G. Netting neutrophils are major inducers of type I IFN production in paediatric systemic lupus erythematosus. Science Translational Medicine 2011

4. Lande R. Plasmacytoid dendritic cells sense self-DNA coupled with antimicrobial peptide. Nature 2007.

Acknowledgements We thank Dr. Ronnblom and Dr Eloranta for providing us with immune complexes material for our scientific project.

Disclosure of interest None declared

\section{NIK-IKK COMPLEX CONTROLS NF-KB-DEPENDENT INFLAMMATORY ACTIVATION OF THE ENDOTHELIUM IN RESPONSE TO LTBR LIGATION}

${ }^{1} \mathrm{P}$ Kucharzewska, ${ }^{2} \mathrm{KCM}$ Jeucken*, ${ }^{2} \mathrm{CX}$ Maracle, ${ }^{2} \mathrm{JP}$ van Hamburg, ${ }^{1} \mathrm{H}$ Olsson, ${ }^{2} \mathrm{SW}$ Tas. ${ }^{1}$ Department of Bioscience, RIA Imed, AstraZeneca, Gothenburg, Sweden; ${ }^{2}$ Experimental Immunology/Clinical Immunology and Rheumatology, AMC Amsterdam, Amsterdam, Netherlands

\subsection{6/annrheumdis-2018-EWRR2018.4}

Introduction Sites of chronic inflammation, such as rheumatoid arthritis (RA) synovial tissue, often contain tertiary lymphoid structures with high endothelial venules (HEV). Ligation of the lymphotoxin (LT)- $\beta$ receptor (LT $\beta$ R) results in activation of canonical and NF-kB-Inducing Kinase (NIK)-dependent noncanonical NF- $\kappa \mathrm{B}$ signalling in endothelial cells (EC), leading to HEV development. However, the relative contribution of the individual NF- $\mathrm{B}$ pathways to inflammatory activation of EC is largely elusive.

Objectives To identify the molecular pathways by which LT $\beta R$ drives inflammatory activation of EC to promote interaction with leukocytes.

Methods Primary human EC were treated with LT $\beta$ or LIGHT to activate LT $\beta R$, followed by analysis of downstream NF- $\kappa B$ signalling pathways and expression of adhesion molecules and inflammatory cytokines. To repress canonical NF- $\kappa B$ signalling pathway, a small molecule inhibitor of IKK $\beta$ was used, and noncanonical NF- $\kappa \mathrm{B}$ signalling was repressed with siRNAs targeting NFKB2. The role of NIK in LT $\beta$ R signalling was investigated using small molecule inhibitors and siRNAs targeting NIK, as well as adenoviral overexpression of NIK. The role of NF- $\kappa B$ signalling in RA was measured by stimulating EC with RA synovial fluid (RASF) followed by analysis of inflammatory mediators.

Results LT $\beta$ R-triggering in EC resulted in activation of both canonical and noncanonical NF- $\mathrm{KB}$ signalling, and induced inflammatory cytokine expression and immune cell adhesion. IKK $\beta$ inhibition repressed LT $\beta R$-induced inflammatory activation of EC, indicating that this process was mediated through canonical NF- $\mathrm{BB}$ signalling. Interestingly, inactivation of NIK also decreased LT $\beta$ R-induced expression of inflammatory cytokines and leukocyte-adhesion, but silencing of NFKB2 had no apparent effect. Further analyses, including silencing and overexpression of NIK, demonstrated a clear role for NIK in activation of the canonical NF- $\kappa \mathrm{B}$ pathway by amplifying IKK complex activity. RASF stimulation of EC resulted in 